\title{
Intravenous oxycodone compared to morphine for post-operative opioid-related adverse events in opioid naïve patients: a prospective randomized controlled trial
}

philippe cuvillon ( $\triangle$ philippe.cuvillon@chu-nimes.fr )

Hopital Universitaire Caremeau https://orcid.org/0000-0002-4424-4280

Sandrine Alonso

Hopital Universitaire Caremeau

Joel L'Hermite

Hopital Universitaire Caremeau

Vanessa Reubrecht

Assistance Publique - Hopitaux de Paris

Lana Zoric

Hopital Universitaire Caremeau

Nathalie Vialles

Hopital Universitaire Caremeau

Jean Luc Faillie

Centre Hospitalier Regional Universitaire de Montpellier

Pascal Kouyoumdjian

Hopital Universitaire Caremeau

Christope Boisson

Hopital Universitaire Caremeau

Mathieu Raux

Assistance Publique - Hopitaux de Paris

Olivier Langeron

Assistance Publique - Hopitaux de Paris

Research article

Keywords: oxycodone, morphine, opioid, surgery, adverse event, safety

Posted Date: November 2nd, 2019

DOI: https://doi.org/10.21203/rs.2.16541/v1 
License: (c) (i) This work is licensed under a Creative Commons Attribution 4.0 International License. Read Full License 


\section{Abstract}

Bacground As intravenous oxycodone is as potent as morphine for pain relief, the purpose of this study was to assess opioid-related adverse events (ORAEs) of oxycodone vs morphine $(1 \mathrm{mg} / 1 \mathrm{mg})$ in opioid naïve patients after general anesthesia and surgery.

Methods Patients schedulled for total hip arthroplasty under general anesthesia were randomized in a 1:1 ratio to receive oxycodone or morphine (titration in PACU followed by intravenous PCA: bolus $1 \mathrm{~mL}$, lockout time $7 \mathrm{~min}$ ). Primary endpoints was number of patients with $\geq 1$ ORAEs at $\mathrm{H} 24$ (at least one of the following complications: nausea, vomiting, respiratory depression, pruritus (itch), urinary retention requiring evacuation, allergy (skin reaction), hallucination (perception without object)). Patients were followed up to 4 months.

Results Among 241 patients included in the ITT analysis, there was no difference in patient characteristics. At $\mathrm{H} 24$, there were 55 patients with at least one ORAEs in oxycodone group versus 46 in morphine group ( $48 \%$ vs $40 \%, p=0.19$; relative risk RR (oxycodone vs morphine) $=1.22[0.91 ; 1.63]$ ). Oxycodone vs Morphine requirements were respectively: 6 [0-11] vs 8 [0-12] mg for titration in PACU $(p=0.06)$ and 15 [8-26] vs 8 [5-16] mg during PCA period at H24 ( $p=0.001)$. The total dose of morphine administered within the first $24 \mathrm{~h}$ was 22 [12-37] vs 19 [11-28]mg for oxycodone $(p=0.048)$. Pain scores (rest, movement: $\mathrm{H} 0-\mathrm{H} 48$ ) and neuropathic pain score after 4 months were similar in both groups.

Conclusion Even if lower doses of oxycodone were required for PACU titration and over the first $24 \mathrm{~h}$, it did not lead to less ORAEs.

\section{Background}

The introduction of multimodal analgesia (including anti-inflammatory drugs), regional anaesthesia and local anaesthetics infiltration have been recently developed to enhance post-operative rehabilitation, pain relief and to reduce opioid consumption [1-4].Despite increased clinical research on recent advances in non-opioid analgesic, many patients still complain about severe post-operative pain and need an initiation of opioid (intravenous i.v. or oral) in PACU (postoperative care unit) nor during the first postoperative days [5-7].

When administered i.v. as a relief medication for acute post-operative pain, morphine has currently become the benchmark for i.v. pain management because it provides rapid and effective analgesia [5-7]. However, since the 1990s, oxycodone has been proposed as an alternative to morphine, because by acting on the $\mu 1$-receptors and on the $\mathrm{k}-2$-opioid agonist, it leads to a better anti nociceptive effect for post-operative pain relief or cancer-related pain [8-14]. Oral studies have shown that oxycodone was associated with better pain control, less serious opioid-related adverse events (ORAEs) and a faster onset of action due to a better pharmacokinetic profile: higher oral bioavailability and lower plasma variability [15-19]. However, controversies have arisen as a result of recent clinical studies and meta-analysis that have shown that oxycodone does not reduce adverse events and pain relief, not to mention that appetite 
for oxycodone itself is decreasing, due to concerns about addiction and the bad reputation that this drug has acquired in opiate-related crises [11, 13, 14, 19-23].

For iv use, studies have shown that the potency ratio of i.v. oxycodone to morphine was 1:1 or 1:2, but no large clinical studies have clearly demonstrated the benefit/risk (ORAEs) of i.v. oxycodone versus morphine $[16,24]$. Also, we conducted this triple blinded study to assess, between patients who received i.v. oxycodone vs morphine after surgery and anesthesia, the proportion of patients with ORAEs. We hypothesized that oxycodone could reduce significantly the number of patient with ORAEs at h24 (primary outcome) and improve pain relief (secondary outcome).

\section{Methods}

\section{Institutional Human Committee, consent and setting}

According to the French law, the present study was approved by the institutional human investigationcommittee (Comité de Protection des Personnes, CPP, Nîmes, France, EudraCT: 2011004140-22, Chaiperson Prof T. Lavabre-Bertrand) on 7 January 2012 and was registered before started on ClinicalTrials.gov (NCT 01536301) [25]. Study was conducted in two French University centres (APHP, La Pitié-Salpêtrière Paris, France; Hôpital Carémeau, Nîmes, France). Unblinding of the investigators performing the analysis occurred after patient enrolment was completed and the dataset was analyzed. The CONSORT (Consolidated Standards of Reporting Trials) recommendations for reporting randomized trial were followed.

Written informed consent was obtained from all participants before inclusion.

\section{Study design and Patients}

This was a prospective, randomized, triple blinded (Participant, Investigator, Outcomes Assessor), controlled trial (1:1 ratio) double center control trial. Patients $>18 \mathrm{yrs}$ scheduled for unilateral elective total hip arthroplasty (posterolateral surgical approach) under general anaesthesia and opioid naïve were eligible and approached by the surgeon or the investigators. Excluded criteria were: refusal to participate, age $>80 \mathrm{yrs}$, weight $<50$ or $>100 \mathrm{~kg}$, emergency or bilateral surgery including hip fracture, any regional anaesthesia or analgesia (including: wound infiltration, peripheral nerve block, epidural or spinal injection), cognitive disorders (delirium, dementia...), pregnancy, patients with alcohol or drugs abuse, uncontrolled epilepsy, patients unlikely to be fully cooperative during the study, participation in another study within the previous 30 days. Patients with chronic pain and/or reporting any allergy or contraindication to study drug (creatinine clearance $<50 \mathrm{~mL} \mathrm{~min}{ }^{-1}$, Cockroft formula), hepatic insufficiency (transaminases and/or alkaline phosphatases $>3$ times of upper normal value, and/or prothrombin time $<70 \%$ of control), acute or chronic respiratory insufficiency (Spo2< $94 \%$ in ambiant air), porphyria, intracranial hypertension, or ileus were not included. Finally, patient already taking narcotics or 
opioid, opiate agonist (codeine, dextromoramide, dihydrocodeine, oxycodone per os, tramadol, morphinelike antitussive...) or agonist-antagonists (buprenorphine, nalbuphine, pentazocine) were not included.

\section{Study Drug, randomization and blinding}

Patients were randomly assigned to the oxycodone group or morphine group using computer-generated random numbers created by the study statistician using a 1:1 randomization ratio. Group allocations were concealed in sequentially numbered opaque envelopes, which were opened by the research nurse just after the start of surgery. Randomization for the two centers were performed using blocks of randomization (8 patients per block). Pharmacy personnel not involved in clinical care of the patient prepared drug solutions. The computer and send to the nurse not involved in the care of the patient generated electronic message. The morphine and oxycodone vials were similar, allowing a double-blinded design. In the case of a serious adverse event, the double-blind could be removed. Patients, surgeons, attending anaesthetists, nurses and investigators were blinded to group assignment until data analysis.

Drug solutions were prepared using:

- $95 \mathrm{~mL}$ of saline solution

- $5 \mathrm{~mL}$ solution of study drug (20 mg mL-1): $100 \mathrm{mg}$ of oxycodone or morphine

From the $100 \mathrm{~mL}$ drug solutions ( $1 \mathrm{mg}: 1 \mathrm{~mL}$ ), $20 \mathrm{~mL}$ of the solution were transferred in $20 \mathrm{~mL}$ syringe and used for titration in PACU. The remaining $80 \mathrm{~mL}$ were used for the patient-controlled analgesia (Gemstar, Abbott Laboratories, Abbott Park, IL, USA) for a 48-h period. The settings for PCA were boluses of $1 \mathrm{~mL}$ with a lockout interval of 7 min without infusion limit. No basal infusion was used.

\section{Study drug administration and background analgesic protocol}

Thirty minutes before the end of the surgery, all patients received i.v. $1 \mathrm{~g}$ paracetamol over 15 minutes, $100 \mathrm{mg}$ ketoprofene and $20 \mathrm{mg}$ nefopam. First two were continued for 48 hours (at 6-h intervals for paracetamol and 12-h for ketoprofene).

In the PACU, patients experiencing pain with a numeric pain rating scale (NRS, $0-10)>3$ were given an i.v. manual titration of $3 \mathrm{~mL}$ of study drug $(2 \mathrm{~mL}$ when body weight $<60 \mathrm{~kg})$ at 5 -min intervals until a NRS score $\leq 3$ was obtained according to a protocol already used routinely in our PACU. All nurses in the PACU had been trained to assess pain using unidimensional scales and to perform opioid titration. At this time, the i.v. PCA with study drug $(80 \mathrm{~mL})$ was connected to the patient for $48 \mathrm{~h}$.

Drug titration was stopped if the patient had a respiratory rate lower than 12 breaths $\min ^{-1}$ and/or an SpO2 lower than $94 \%$ and/or experienced a serious adverse event related to opioid administration (allergy 
with cutaneous rash, vomiting, severe pruritus) and also if the patient was asleep. In case of severe ventilatory depression (respiratory rate, $\mathrm{RR}<10$ breaths $\mathrm{min}^{-1}$ ) an intravenous bolus of $0.04 \mathrm{mg}$ naloxone was administered until RR was greater than 12 breaths $\mathrm{min}^{-1}$, and this was defined as a severe ORAEs.

\section{General anaesthesia and perioperative management}

In both groups, an oral premedication was given $1 \mathrm{~h}$ before surgery (hydroxyzine $1 \mathrm{mg} \mathrm{kg}^{-1}$ ). General anaesthesia was induced with propofol $\left(2-3 \mathrm{mg} \mathrm{kg}^{-1}\right)$, sufentanil $\left(0.3 \mu \mathrm{g} \mathrm{kg}^{-1}\right)$, and cisatracrium (0.3$\left.0.5 \mathrm{mg} \mathrm{kg}^{-1}\right)$. Airway was maintained with tracheal tube and the lungs were ventilated with a mix of oxygen-nitrous oxide (50/50). Tidal volume and respiratory rate were set to maintain end tidal volume $\mathrm{CO}_{2}$ between 35 and $40 \mathrm{mmHg}$. A $5-\mathrm{cm} \mathrm{H}_{2} \mathrm{O}$ positive end expiratory pressure was set. Anaesthesia was maintained with sevoflurane $1-2 \%$ and additional intravenous sufentanil $(5-10 \mu \mathrm{g})$ upon request. I. V. fluid administration was ringer lactate $<10 \mathrm{~mL} \mathrm{~kg}^{-1} \mathrm{~h}^{-1}$. Urinary intra-operative catheter was not placed.

At the end of surgery, patients were extubated and transferred to the PACU. Prevention of nausea or vomiting was based on Apfel score. For patients with score 1 or 2, i.v. dexamethasone $0.1 \mathrm{mg} \cdot \mathrm{kg}^{-1}$ was injected after the induction of anaesthesia, followed with i.v. 4-8 $\mathrm{mg}$ ondansetron for score $>2$. The Aldrete's scoring system was used for determining when the patient can be discharged (score: 0-10: a score 9 was required for discharge).

Over the first $48 \mathrm{~h}$ post-operative period, patients in both groups were managed similarly and standardized: early oral intake and post-operative rehabilitation, i.v. ondansetron $(4 \mathrm{mg})$ was injected in case of nausea or vomiting. Patients were discharged from the surgical ward at the discretion of the surgeon.

\section{Clinical assessment}

Preoperative evaluation was performed the day before surgery, including: NRS pain score at rest at walking (0-10), DN4 score (0-10), walking ability $(\mathrm{m})$, medical diseases, physiological parameters (blood pressure, heart and respiratory rate, pulse oxymetry), biological variables (Haemoglobin, creatinine clearance). Pain intensity and adverse events were assessed in PACU and every $6 \mathrm{~h}$ over the study period $(48 \mathrm{~h})$. The nurse staff evaluated patient pain intensity using a NRS at rest and on movement (hip mobilization). When the patient was asleep, no attempt was made to wake him up, and the patient was considered as having analgesia and a score of $0 \mathrm{~mm}$ was assigned to the patient. Total drug administration in PACU and over the $48 \mathrm{~h}$ were recorded.

ORAEs. arising from the analgesic protocol were systematically assessed in PACU and surgical ward by nurses in charge of the patient (blinded to group assignment): nausea, vomiting, drowsiness, dizziness, headache, sweating, itching, confusion/hallucination, pruritus, sedation, arterial hypotension (mean arterial pressure $<80 \mathrm{mmHg}$ ), respiratory depression. Theses outcomes were viewed as binary (Yes/No), 
every $6 \mathrm{~h}$ after PACU. Arterial oxygen saturation (pulse oximetry), heart and respiratory rates and blood pressure was recorded every $6 \mathrm{~h}$. Desaturation was considered pulse oxygen saturation $<94 \%$. A respiratory rate $<10$ breaths/min was consideredas respiratory depression. Heart rate $>120 \mathrm{bpm}$ and $<50$ bpm defined tachycardia and bradycardia, respectively. Sedation was defined by a Ramsay scale $>2$.

After 4 months, all patients were called and questioned for pain, DN4 score, satisfaction about pain after surgery (using a scale: 0-100) and other adverse events medical or surgical complications [26].

\section{Endpoints}

The main end point was the rate of opioid-related adverse effects from start of titration to $24 \mathrm{~h}$. As previously described, the following ORAEs were the presence/absence of at least one of the following complications: nausea, vomiting, respiratory depression, pruritus (itch), urinary retention requiring evacuation (spontaneous voiding impossible despite bladder volume $>400 \mathrm{ml}$ measured by ultrasound), allergy (skin reaction), hallucination (perception without object). Ventilator depression was defined as RR below $10 \mathrm{~min}^{-1}$ or need for naloxone administration. Sedation was defined as a Ramsay score above 2 but was not considered as an ORAEs

The secondary efficacy end-points were: the time to achieve pain relief in the PACU, the number of patients with post-operative severe pain, the number of patients who required titration, the duration of stay in the PACU period, the consumption of opioid during the PACU and PCA periods, the number of demands for opioid and the number of boluses received during the PCA period, the total dose administered over $24 \mathrm{~h}$, the VAS during the PACU and the PCA periods, and the patient satisfaction assessment.

\section{Sample Size Calculation}

The sample size was calculated based on the ability to detect a significant difference in adverse events between oxycodone versus morphine groups with a difference in the ORAEs occurrence of $50 \%$ (32\% vs $16 \%$ ) with $80 \%$ power, based on previous study with morphine. ${ }^{1-10}$ The level of significance was set at two-sided $=0.05$ to support the hypothesis that ORAEs oxycodone group were different than that in the morphine group. Using these assumptions, we calculated a sample size of 222 patients (111 patients per group). We increased the number to 246 patients (123 per group) to account for $10 \%$ loss to follow-up.

\section{Statistical analysis}

Statistical analysis was conducted using SAS (9.4; SAS Inc., Cary NC). An intention-to-treat analysis was performed without any interim analysis. Statistical results were expressed with mean (SD) or median [25-75 IQ] according to the distribution. The numbers and associated percentages were given for categorical variables. Comparisonsof continuous variables between the groups were performedusing a 
student's $t$ test or Wilcoxon-Mann-Whitney test according to the distribution. Categorical variables werecompared between groups by $X^{2}$ or Fisher's exacttest.

All statistical tests were conducted as 0.05 two-sided tests.

\section{Results}

\section{Population of the study}

From June 2012 to July 2016, 623 patients scheduled for primary unilateral total hip arthrosplasty were screened and 246 patients were randomized (Fig.1). After randomization, 5 patients were excluded (4 for protocol violation, one because surgery was cancelled). These patients were not included in the analysis $(n=241)$. Three patients discontinued the treatment. At 4 months, 102 and 106 patients in oxycodone and morphine group completed their end-of-study visit, respectively.

Baseline characteristics, preoperative pain, physiological and biological parameters, surgery and general anaesthesia duration were similar in both groups (Table 1). Premedication (64 vs 70 patients), total intra operative sufentanil injection ( 40 30-45 vs 3530-45 g), median perioperative i.v. fluid (1225 vs $1000 \mathrm{~mL}$ ) and number of patients requiring red blood transfusion ( $1 v s 2)$ were well balanced between groups. Moreover, the number of patients receiving iv ondansetron ( 6 vs 3) or i.v. dexamethasone (72 vs 65) during general anaesthesia was similar.

\section{Primary outcome (Table 2)}

The number of patients with at least one ORAEs was similar in both group: $\mathrm{n}=55$ (48\%) (oxycodone group) vs $\mathrm{n}=46(40 \%)$ (morphine group) $(P=0.19)$ with a relative risk RR (oxycodone vs morphine) $=$ $1.22[0.91 ; 1.63]$. Nausea and vomiting were the main ORAEs in $23 \%$ patients in both groups in PACU (Table 2). Respiratory depression was the first ORAEs in PACU (all patients: $21 \%$ ), without any respiratory complication after PACU. Urinary retention was the first ORAEs in both groups after PACU discharge until H24 (all patients: $15 \%$ ) (Table 2).

\section{Secondary outcomes}

The initial NRS pain in PACU (4 0-6 in oxycodone group vs 40-7 in morphine group) and the number of patients requiring titration (NRS pain $>3 / 10$ ) were similar in both groups (Table 3 ). Number of boluses to obtain a NRS pain $<3 / 10$ was reduced in oxycodone group as compared with morphine group $(P=0.03)$, but no difference was observed for the time of titration and the PACU time discharge (Table 3). Time $<45$ min to get a NRS pain $<3 / 10$ was recorded for 76 (95\%) patients in oxycodone group vs $75(85 \%)$ in morphine group $(P=0.036)$ with a RR oxycodone vs morphine $=0.34[0.12 ; 0.99]$. 
The number of ORAEs (Table 2), the number of sedated patients (1 vs 1 ), and the number of patients requiring termination of morphine titration ( 2 vs. 3 [NS] were similar between groups. Morphine or oxycodone requirements during the PCA period was less in morphine group, but the total dose of both drugs administered within the first $24 \mathrm{~h}$ were significantly different between groups (Table 3 ) Over the study period, similar NRS pain at rest or movement were recorded for both groups, with a median score < $3 / 10$ at each time $(\mathrm{H} 6,12,18,24,30,36,42,48)$ (Figure $2 A$ and $2 B$ ). Patient satisfaction was not different in both groups [77 (26) $\mathrm{mm}$ in morphine group vs 73 (27) $\mathrm{mm}$ in oxycodone group, NS] No difference was observed at the end of the follow up period (4 month) regarding DN4 scores ( $p=0.78)$, median VAS pain score at movement $(P=0.67)$ and satisfaction $(P=0.47)$ (table 3$)$. The number of patients included in this analysis was 51 in oxycodone group and 53 in morphine group.

\section{Discussion}

This prospective triple blinded study demonstrated that i.v. oxycodoene did not lead to less opioid-related ORAEs in the first $24 \mathrm{~h}$ after major othopedic surgery as compared with morphine. Post-operative pain relief was similar between the two drugs, with no benefit for chronic pain.

\section{PACU period}

In PACU, i.v. morphine titration demonstrated effectiveness on post-operative pain relief and is widely recommended when NRS pain score $>3 / 10[5,6,21]$. Studies conducted in major orthopedic surgery or comparing young patients with elderly patients have shown that i.v. morphine administration every 5 min with an unlimited number of 2- or 3-mg boluses provided the best and fastest analgesia [5,6]. In the present study, we demonstrated that with a similar administration regimen, the morphine group tended to experience more pain in the PACU and required more adjustments $(P=0.03)$ (Table 3, Fig 2$)$. This is an important issue, because oxycodone has been shown to have a faster onset of action than morphine, allowing for better analgesia when pain is severe and rapid adjustment is required. However, our study clearly demonstrated a lack of clinical difference between both drugs when opioid are required in PACU because the difference was only 1 bolus (ie 2 to $3 \mathrm{mg}$ ). Moreover, the number of patients who had the full dose of maximal i.v. titration was similar between the two groups. For primary end point, the rate of ORAEs reported in PACU were similar. The main reason for this lack of difference is the similarity of the adjustment dosing regimen between the two groups with close final doses.

Urinary retention was the most frequent ORAEs reported in PACU, largely linked to the duration of general anaesthesia and intraoperative fluid administration. This event may vary depending on the type of surgery. Doses of sufentanil and sevoflurane influence the level of PONV. In our study, both groups ultimately received equivalent doses and finally nausea or vomitng were similar between groups and with studies already published elsewhere $[15,19,27,28]$.

The patients in this study did not receive the optimal multimodal analgesic regimen, in order to minimize the need of post-operative rescue opioids, but this was designed for this study. The optimal regimen will 
include: spinal anaesthesia (instead of general), perioperative analgesic dose of glucocorticoid (most patients in the study received dexamethasone for nausea prophylaxis, but probably a suboptimal dose), the use of local anaesthesia wound infiltration (only in the wounds or better, as LIA protocol).

\section{PCA period}

For the PCA period ( $0-24 \mathrm{~h})$, this study did not demonstrate any benefit in favor of oxycodone as regards the incidence of side effects or regimen of drug administration.

The incidence of nausea and vomiting were similar between the two groups, $<10 \%$ (Table 3 ). This rate appeared to be low but probably related to the systematic prevention of nausea used in this study (see method). With oxycodone, the incidence of PONV in the literature vary according to dose-related studies and route of administration (p.o., i.v., s.c...) $[15-19,23]$. In fact, no study appears to ultimately demonstrate superiority to oxycodone vs morphine. Using morphine doses of $45 \mathrm{ug} \mathrm{kg}^{-1}$ or oxycodone 30 $\mu \mathrm{g} \mathrm{kg}^{-1}$ as i.v. bolus, Silvasti et al. found no difference at $\mathrm{H} 24$ after breast surgery on a weak collective (50 patients) [19]. Performing plasma assays, authors demonstrated the equipotent effect of both drugs, so that morphine and oxycodone appeared to be equipotent. After laparoscopic supracervical hysterectomy, Kim et al. found a higher rate of nausea with oxycodone of $48 \%$ at $\mathrm{H} 24$ (vs $14 \%$ in fentanyl group) [29]. In this study, the percentage of patients experienced at least 1 adverse event for drowsiness and dizziness were higher in oxycodone group (respectively, 3 and $25 \%$ ) than in fentanyl group (respectively, 25 and $67 \%$ ) [29]. Conversely, no significant difference between the 2 groups were noted for ORAEs in a similar study [28].

Regarding opioid-induced excessive sedation after hysterectomy (under general anaesthesia), Lenz et al demonstrate a lower rate in favour of oxycodone [22]. But the accumulated $24 \mathrm{~h}$ oxycodone consumption was significantly less compared with the accumulated morphine consumption (13 mg vs $22 \mathrm{mg}, P$ $<0.001)$. In our study, no differences were observed with respect to sedation or respiratory depression. Ultimately, the literature review does not appear to show a clear benefit for oxycodone in reducing the incidence of adverse events. This is demonstrate in our study which is the first one based on this main objective. These results corroborate the latest meta-analyze performed in oncology that showed no benefit to oxycodone [11-13].

The value of oxycodone in reducing postoperative neuropathic or chronic pain has remained debated. This study showed no difference at 4 months when patients were called and the DN4 score was analyzed. Surgery with more frequent chronic pain could be evaluated to further analyze this effect.

\section{Limite biais}

The limitations and biases of our study should be noted. First, the surgical model chosen (intermediate pain). Different results could be noted with other surgeries and different selected populations (bias 
related to individual variations in psychological disorders or pain sensitivity). The incidence of pain and adjustment doses vary according to gender, age and type of surgery (anterior, posterolateral...). Our results are only valid for a relatively healthy patient population (not too old, not too fat, no kidney failure, no use of preoperative opioids) and for this procedure only. Moreover, the study was performed at two different centers, which may weaken the power of the data as many subtle issues (surgeons, anaesthesiologists, everyday practice, patient recruitment), but intra and post-operative care were standardized. Secondly, the ratio chosen $1 / 1$ is always a source of discussion and disagreement between the experts due to peak effect $(1 / 1,1 / 1.5,1 / 2)$ [30]. To reduce external bias, we decided to work on a similarity of all volumes and doses with an extending evaluation at $\mathrm{H} 24$ (rather than in PACU or H12). Composites (results) are often used to reduce sample size, but can cause particular problems when the components are of different importance to patients, occur with different frequencies and are affected to a different degree by the intervention. The high and similar frequency of ORAEs in our study corresponds to this ideal of choice. Finally, the fact that this study excluded opioid patients prior to surgery is an important nuance that has a substantial impact on the generalizability of the study, as many patients who underwent surgery for painful reasons are already receiving opioid treatment.

\section{Conclusion}

In conclusion, for clinical purposes, oxycodone or morphine can be used as first-line i.v. opioids for relief of surgery pain in adults. Oxycodone induced faster rescue analgeisa in PACU, but did not demonstrate less ORAEs at $\mathrm{H} 24$ nor better pain relief.

\section{List Of Abbreviations}

i.v.: intravenous

NRS: Numeric Rating Scale

ORAEs: Opioid-Related Adverse Events

PACU: Postoperative Care Unit

PCA: Patient Controlled Analgesia

RR: Respiratory Rate

\section{Declarations}

- Ethics approval and consent to participate: the present study was approved by the institutional human investigationcommittee (Comité de Protection des Personnes, CPP, Nimes, France, EudraCT: 2011004140-22, Chaiperson Prof T. Lavabre-Bertrand) on 7 January 2012 and was registered before started 
on ClinicalTrials.gov (NCT 01536301). A written informed consent was obtained from all participants before inclusion.

- Consent for publication: not applicable

- Availability of data and materials: The datasets used and/or analysed during the current study are available from the corresponding author on reasonable request.

- Competing interests: The authors declare that they have no competing interests

- Funding: this work was only supported by our institutional sources (Carémau Hospital, Nîmes, France) and funding were only used for the payment of IRB and to purchase study drugs.

- Authors' contributions: all authors have read, revised and approved the final manuscript. $P C, M R$ and $O L$ were responsible for the conception and design of the study, for the coordination of the centres and data analysis. PC and JYL writed the manuscript. SA was responsible for data and statistical analysis. $L Z, V R$, $C B, N V$ and $J L H$ were responsible for the patient recruitment and for anesthetizing the patients.

- Acknowledgements: Ariane Lannelongue for technical help (English spelling and Figure)

\section{References}

1 Cuvillon P, Zoric L, Demattei C et al. Opioid-sparing effect of nefopam in combination with paracetamol after major abdominal surgery: a randomized double-blind study. Minerva Anestesio/ 2017; 83: 914-920.

2 Kehlet $\mathrm{H}$. Accelerated Recovery after Surgery: a continuous multidisciplinary challenge. Anesthesiology 2015; 123: 1219-20.

3 Richebé P, Capdevila X, Rivat C. Persistent postsurgical pain. Pathophysiology and preventive pharmacologic considerations. Anesthesiology 2018; 129: 590-607.

4 Zoric L, Cuvillon P, Alonso S et al. Single-shot intraoperative local anaesthetic infiltration does not reduce morphine consumption after total hip arthroplasty: a double-blinded placebo-controlled randomized study. Br J Anaesth2014; 112: 722-8.

5 Aubrun F, Monsel S, Langeron $\mathrm{O}$, Coriat P, Riou B. Postoperative titration of intravenous morphine in the elderly patient. Anesthesiology 2002; 96: 17-23.

6 Aubrun F, Langeron O, Quesnel C, Coriat P, Riou B. Relationships between measurement of pain using visual analog score and morphine requirements during postoperative intravenous morphine titration. Anesthesiology 2003; 98: 1415-21.

7 Pergolizzi JV Jr, Seow-Choen F, Wexner SD, Zampogna G, Raffa RB, Taylor R Jr. Perspectives on Intravenous Oxycodone for Control of Postoperative Pain. Pain Pract 2016; 16: 924-34. 
8 Nozaki C, Kamei J. Involvement of mu1-opioid receptor on oxycodone-induced antinociception in diabetic mice. Eur J Pharmacol 2007; 560: 160-2

9 Ross FB, Smith MT. The intrinsic antinociceptive effects of oxycodone appear to be kappa-opioid receptor mediated. Pain 1997; 73: 151-7.

10 Dieterich M, Müller-Jordan K, Stubert J, Kundt G, Wagner K, Gerber B. Pain management after cesarean: a randomized controlled trial of oxycodone versus intravenous piritramide. Arch Gynecol Obstet 2012; 286: 859-65.

11 Gaskell H, Derry S, Stannard C, Moore RA. Oxycodone for neuropathic pain in adults. Cochrane Database Syst Rev 2016; 7: CD010692.

12 Jiang Z, Zhou G, Song Q, Bao C, Wang H, Chen Z. Effect of Intravenous Oxycodone in combination with different doses of dexmedetomdine on sleep quality and visceral pain in patients after abdominal surgery: a randomized study. Clin J Pain 2018; 34: 1126-1132.

13 Lee $\mathrm{KH}$, Kang JH, Oh HS et al. Intravenous Oxycodone versus Intravenous Morphine in Cancer Pain: A Randomized, Open-Label, Parallel-Group, Active-Control Study. Pain Res Manag 2017; 2017: 9741729.

14 Schmidt-Hansen M, Bennett MI, Arnold S, Bromham N, Hilgart JS. Oxycodone for cancer-related pain. Cochrane Database Syst Rev 2017; 8: CD003870.

15 Cheung CW, Ching Wong SS, Qiu Q, Wang X. Oral Oxycodone for acute postoperative pain: A review of clinical trials. Pain Physician 2017; 20: SE33-SE52.

16 Liukas A, Kuusniemi K, Aantaa R et al. Elimination of intravenous oxycodone in the elderly: a pharmacokinetic study in postoperative orthopaedic patients of different age groups. Drugs Aging 2011; 28: $41-50$.

$17 \mathrm{Nie} J$ J, Sun S, Huang SQ. Effect of oxycodone patient controlled intravenous analgesia after cesarean section: a randomized controlled study. J Pain Res 2017; 10: 2649-2655.

18 Olczak B, Kowalski G, Leppert W, Zaporowska-Stachowiak I, Wieczorowska-Tobis K. J. Analgesic efficacy, adverse effects, and safety of oxycodone administered as continuous intravenous infusion in patients after total hip arthroplasty. Pain Res 2017; 10: 1027-1032.

19 Silvasti M, Rosenberg P, Seppälä T, Svartling N, Pitkänen M. Comparison of analgesic efficacy of oxycodone and morphine in postoperative intravenous patient-controlled analgesia. Acta Anaesthesiol Scand 1998; 42: 576-580.

20 Abou hammoud A, Simon N, Urien S, Riou B, Lechat P, Aubrun F. Intravenous morphine titration in immediate postoperative pain management: population kinetic-pharmacodynamic and logistic regression analysis. Pain 2009; 144: 139-146. 
21 Aubrun F, Mazoit JX, Riou B. Postoperative intravenous morphine titration. Br J Anaesth 2012; 108: 193-201.

22 Lenz H, Sandvik L, Qvigstad E, Bjerkelund CE, Raeder J. A Comparison of Intravenous Oxycodone and Intravenous Morphine in Patient-Controlled Postoperative Analgesia After Laparoscopic Hysterectomy. Anesth Analg 2009; 109:1279-83.

23 Rothwell MP, Pearson D, Hunter JD et al. Oral oxycodone offers equivalent analgesia to intravenous patient-controlled analgesia after total hip replacement: a randomized, single-centre, non-blinded, noninferiority study. Br J Anaesth2011; 106: 865-72.

24 Pereira J, Lawlor P, Vigano A, Dorgan M, Bruera E. Equianalgesic dose ratios for opioids. a critical review and proposals for long-term dosing. J Pain Symptom Manage 2001; 22: 672-87.

25 Champigny V, Holmes-LeBlanc S, Pittet JF. New legal framework for human subject research in France: A step forward? Anaesth Crit Care Pain Med 2018; 37: 529-530.

26 Chatila N, Pereira B, Maarrawi J, Dallel R. Validation of a New Arabic Version of the Neuropathic Pain Diagnostic Questionnaire (DN4). Pain Pract2017; 17: 78-87.

27 Biboulet P, Morau D, Aubas, Bringuier-Branchereau S, Capdevila X, Postoperative analgesia after totalhip arthroplasty: comparison of intravenous patient- controlled analgesia with morphine and single injection of femoral nerve or psoas compartment block. A prospective, randomized, double-blind study. Reg Anesth Pain Med 2004; 29: 102-9.

28 Ding Z, Wang K, Wang B, Zhou N, Li H, Yan B. Efficacy and tolerability of oxycodone versus fentanyl for intravenous patient-controlled analgesia after gastrointestinal laparotomy. A prospective, randomized, double-blind study. Medicine (Baltimore) 2016;95: e4943.

29 Kim NS, Lee JS, Park SY et al. Oxycodone versus fentanyl for intravenous patient-controlled analgesia after laparoscopic supracervical hysterectomy: a prospective, randomized, double-blind study. Medicine (Baltimore) 2017; 96(10): e6286.

30 Levy M. Advancement of opioid analgesia with controlled-release oxycodone. European J Pain 2001; Suppl. A: 113-116.

\section{Tables}

Table 1. Characteristics of the patients, surgery and pain at baseline

$\begin{array}{ll}\text { Oxycodone } & \text { Morphine } \\ n=119 & n=119\end{array}$


$\operatorname{Sex}(M, F)$

Age (yr)

$\operatorname{BMI}\left(\mathrm{kg} / \mathrm{m}^{2}\right)$

ASA (1/2/3/4)

Physiological parameters

Systolic Blood Pressure ( $\mathrm{mmHg}$ )

Heart rate

Respiratory rate

Pulse oxymetry (\%)

Preoperative hip pain

NRS pain at rest (0-10)

NRS pain at walking (0-10)

Time since beginning of pain $(y r)$

DN4 scores (0-10)

DN4 >4 (n)

Use of non opiod analgesics (n)

Use of opioid analgesics ( $n$ )
59 / 61

67 [26-82]

27 [24-31]

$32 / 71 / 15 / 1$

140 [130-150]

$70[65-80]$

13 [12-14]

98 [97-99]
$47 / 74$

$70[35-84]$

26 [24-31]

37 / 65 / 19 / 0

138 [130-150]

70 [67-80]

13 [12-14]

98 [97-99]
$3.2 \pm 2.8 \quad 3.3 \pm 2.5$

$6.8 \pm 2.5 \quad 6.0 \pm 2.5$

$2[1-4]$

$2[1-4]$

$1.4 \pm 1.6$

$1.1 \pm 1.3$

12

8

72

70

0

0

38

40

20

22

26

26

14

10

Walking with a cane (n) 
Biologic variables
Hemoglobin, g.dL ${ }^{-1}$
$14.3 \pm 1.3$
$14 \pm 1.1$
Creatinine clearance, $\mathrm{mL} \cdot \mathrm{min}^{-1}$
77 [68-85]
$72[61-84]$

Surgical side (right/left)

$68 / 52$

$68 / 53$

Surgery duration (h)

$1.42[1.02-2.02]$

$1.58[1.05-2.07]$

General anesthesia duration (h)

$2.33[1.83-3.15]$

$2.5[1.92-3.25]$

Time for extubation (min)

14 [8-23]

$16[9-25]$

Time extubation-first NRS evaluation (min)

$11[5-19]$

$10[5-19]$

Data are mean $\pm S D$, medians $\left[25\right.$ th $\left.-75^{\text {th }}\right]$, or number.

ASA: American Society of Anesthesiologists Physical Status Class

BMI : Body Mass Index

F: Female

M: Male

NRS : Numerical Rating Scale

Table 2. Opioid related adverse events (ORAEs)

$$
\begin{array}{lll}
\text { Oxycodone } & \text { Morphine } & \text { P value } \\
(n=119) & (n=119)
\end{array}
$$

\section{Total period}

Number of patients with $\geq 1$ ORAEs

Nausea

Vomiting

Repiratory depression (need $>3 \mathrm{~L} \mathrm{O}_{2} /$ min) $29(26 \%)$
$55(48)$

$17(15)$

$6(5)$

$6(5)$ 


$\begin{array}{lll}\text { Repiratory depression (naloxone injection) } & 0 \\ \text { Urinary Retention } & 22(20 \%) & 14(12 \%) \\ \text { Pruritus } & 0 & 2(2 \%) \\ \text { Allergy } & 1(1 \%) & 1(1 \%) \\ \text { Cognitive disorder } & 1(1 \%) & 4(4 \%)\end{array}$

\section{PACU period}

$\begin{array}{lcc}\text { Number of patients with } \geq 1 \text { ORAEs } & 32(28 \%) & 27(24 \%) \\ \text { Nausea } & 8(7 \%) & 7(6 \%) \\ \text { Vomiting } & 2(2 \%) & 1(1 \%) \\ \text { Repiratory depression }\left(>3 \mathrm{~L} \mathrm{O}_{2} / \mathrm{min}\right) & 29(26 \%) & 19(17 \%) \\ \text { Repiratory depression (naloxone injection) } & 0 & 0 \\ \text { Urinary Retention } & 2(2 \%) & 1(1 \%) \\ \text { Pruritus } & 0 & 0 \\ \text { Allergy } & 0 & 0 \\ \text { cognitive disorder } & 0 & 1(1 \%)\end{array}$

\section{PCA period until H24}

\begin{tabular}{|c|c|c|}
\hline Number of patients with $\geq 1$ ORAEs & \multicolumn{2}{|c|}{$33(28 \%)$} \\
\hline Nausea & $11(10 \%)$ & $9(8 \%)$ \\
\hline Vomiting & $4(3 \%)$ & $5(4 \%)$ \\
\hline Repiratory depression $\left(3 \mathrm{~L} \mathrm{O}_{2} / \mathrm{min}\right)$ & 0 & $1(1 \%)$ \\
\hline Repiratory depression (naloxone injection) & n) 0 & 0 \\
\hline Urinary Retention & $1(18 \%)$ & $13(11 \%)$ \\
\hline
\end{tabular}




$\begin{array}{llc}\text { Pruritus } & 0 & 2(2 \%) \\ \text { Allergy } & 1(1 \%) & 1(1 \%) \\ \text { Cognitive disorder } & 1(1 \%) & 3(3 \%)\end{array}$

Data are number (\%)

Table 3. Titration, PCA consumption and outcomes

$\begin{array}{lcl}\text { Oxycodone } & \text { Morphine } & \text { P value } \\ (n=119) & (n=119) & \end{array}$

\section{PACU period}

\begin{tabular}{|c|c|c|c|}
\hline Patients requiring titration $(N R S>3 / 10)$ & $80(68)$ & $88(74)$ & 0.30 \\
\hline Number of titration bolus ( $\mathrm{n}$ ) & $3[2-5]$ & $4[3-6]$ & 0.03 \\
\hline Titration dose, mg & $6[0-11]$ & $8[0-12]$ & 0.06 \\
\hline Time to obtain NRS pain $<3 / 10$ (min) & 20 [10-25] & 20 [15-40] & 0.15 \\
\hline Sedated patients(Ramsay score $>2$ ) & $1(0.9)$ & $1(0.9)$ & 1 \\
\hline Time to discharge $(\mathrm{h})$ & $2.0[1.5-2.5]$ & $1.9[1.5-2.6]$ & 0.83 \\
\hline
\end{tabular}

PCA period (after PACU to H24)




$\begin{array}{llll}\text { Total dose of study drug }(\mathrm{mg}) & 22[12-37] \quad 19[11-28] & 0.048\end{array}$

Outcomes after $24 h$

At discharge from hospital

Time to hospital discharge (days) $6[5-7] \quad 6[5-7] \quad 0.12$

Global satisfaction (0-10) $\quad 9[7-10] \quad 9[7-10] \quad 0.88$

After 4 months

$\begin{array}{llll}\text { DN4 score }(0-10) & 0[-1-2] & 0[-1-1] & 0.78\end{array}$

Global satisfaction (0-10) $\quad 9[8-10] \quad 9[7-10] \quad 0.47$

Data are number (\%)

PCA: Patient Controlled Analgesia

Figures 


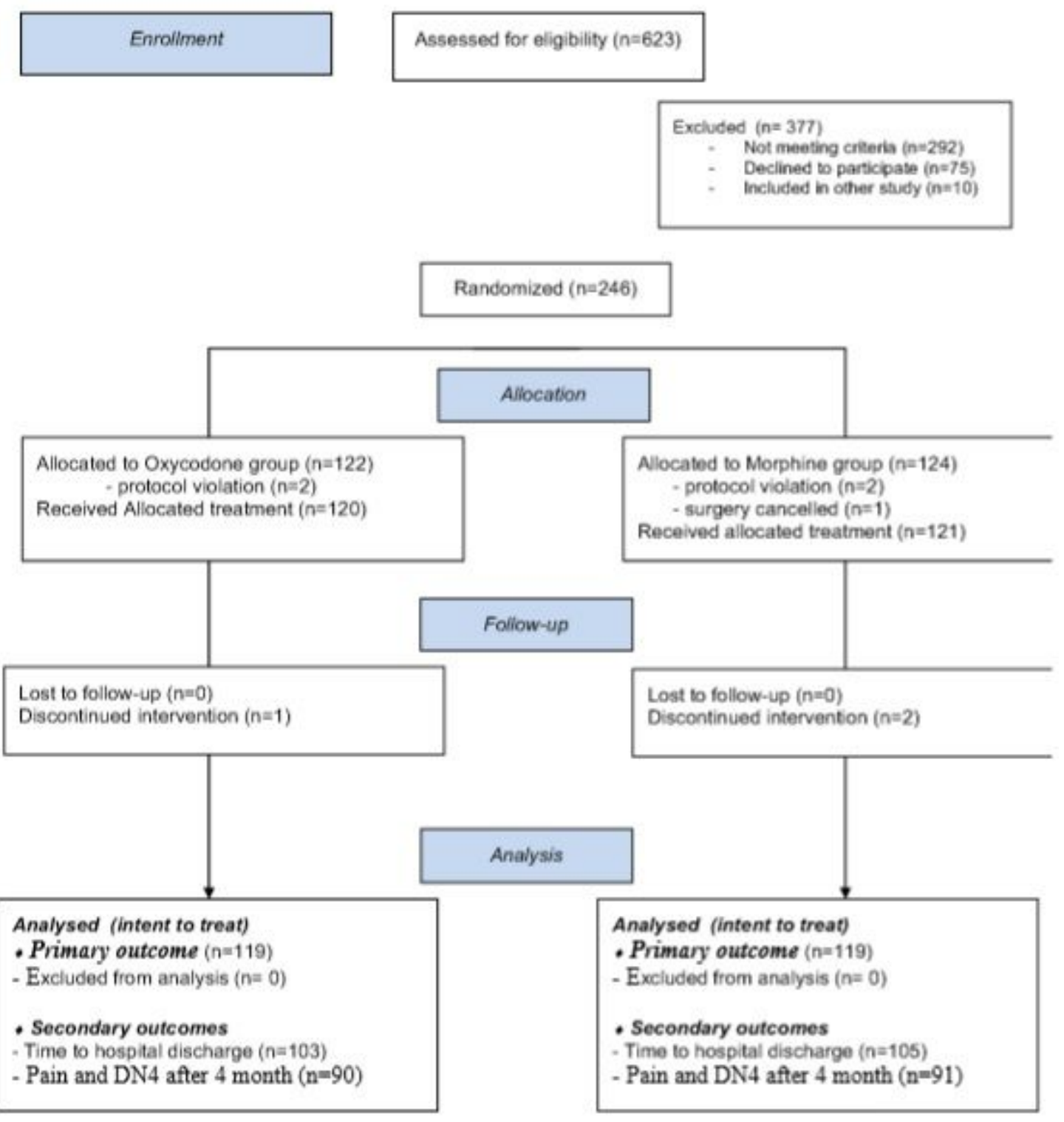

\section{Figure 1}

Flow diagram of the study 

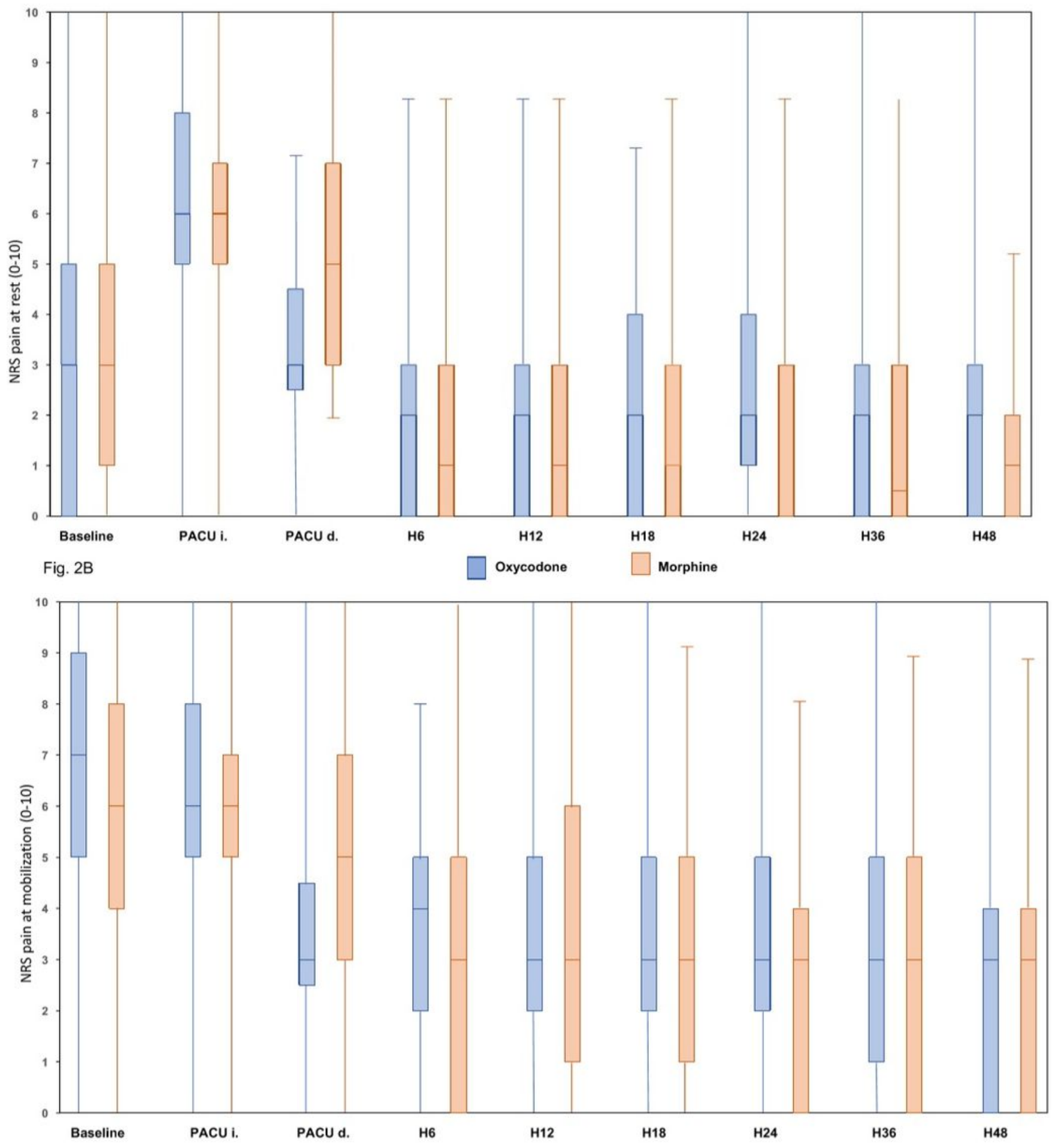

Figure 2

Box and wisker plots showing the median, interquartile range, and range of pain scores at rest (Fig2 A) and at movement (Fig2 B) across time for oxycodone and morphine group. Baseline was performed at inclusion ( $>24 \mathrm{~h}$ and $<6$ weeks before surgery). PACUi: initial scores after extubation and before titration in Post Operative Care Unit. PACUd: scores at discharge. No difference was observed between groups at baseline. ${ }^{*}<0.05$ vs oxycodone 


\section{Supplementary Files}

This is a list of supplementary files associated with this preprint. Click to download.

- CONSORTChecklist3.doc 